\title{
Cognitive impact of neuronal antibodies: encephalitis and beyond
}

\author{
L. L. Gibson' ${ }^{1}$, A. McKeever ${ }^{2}$, E. Coutinho ${ }^{3,4}$, C. Finke $e^{5,6}$ and T. A. Pollak $\mathbb{1}^{7}$
}

\begin{abstract}
Cognitive dysfunction is a common feature of autoimmune encephalitis. Pathogenic neuronal surface antibodies are thought to mediate distinct profiles of cognitive impairment in both the acute and chronic phases of encephalitis. In this review, we describe the cognitive impairment associated with each antibody-mediated syndrome and, using evidence from imaging and animal studies, examine how the nature of the impairment relates to the underlying neuroimmunological and receptor-based mechanisms. Neuronal surface antibodies, particularly serum NMDA receptor antibodies, are also found outside of encephalitis although the clinical significance of this has yet to be fully determined. We discuss evidence highlighting their prevalence, and association with cognitive outcomes, in a number of common disorders including cancer and schizophrenia. We consider mechanisms, including blood-brain barrier dysfunction, which could determine the impact of these antibodies outside encephalitis and account for much of the clinical heterogeneity observed.
\end{abstract}

\section{Introduction}

An expanding array of pathogenic neuronal autoantibodies are being identified, each targeting different neuronal surface antigens and thought to cause distinct and clinically recognisable encephalitic syndromes. These antigenic targets have wide-ranging properties and distributions in the central nervous system but common to almost all autoimmune encephalitides is cognitive dysfunction. The nature of cognitive impairment and associated neuroimaging findings varies between syndromes and gives insight into the antibody-mediated mechanism of action. Neuronal autoantibodies have also been reported-so far mainly in the peripheral blood-in individuals without frank encephalitis. Although their significance outside the encephalitic context is not yet clear, there is a growing body of evidence to suggest these autoantibodies have pathogenic potential even in the absence of the encephalitic syndrome. In this review, we

\footnotetext{
Correspondence: T. A. Pollak (thomas.pollak@kcl.ac.uk)

${ }^{1}$ Department of Old Age Psychiatry, Institute of Psychiatry, Psychology and

Neuroscience, King's College London, London, UK

¿University of Cambridge, Cambridge, UK

Full list of author information is available at the end of the article.
}

outline the cognitive profile of each of the commonest autoantibody-mediated encephalitides and consider the role of neuronal antibodies outside encephalitis. While a treatment of the neurotransmitter basis of cognition is beyond the scope of this review, it is important to note that these autoantibodies largely serve to disrupt the signalling transmission of neurotransmitters such as glutamate and $\gamma$-aminobutyric acid (GABA) which are integral to cognition. Glutamate is a ubiquitously distributed excitatory neurotransmitter that also acts as an intermediary in cerebral metabolism; the ionotropic glutamate-specific N-Methyl-d-aspartate receptor (NMDAR) and $\alpha$-amino-3-hydroxy-5-methyl-4-isoxazolepropionic acid (AMPA) receptors are vital components of long-term potentiation (LTP) and long-term depression, processes understood to be the major synaptic substrates of learning and memory; changes in the neuronal surface density of these receptors therefore have direct effects on neuronal signalling with downstream impact on brain connectivity and cognitive processes. GABA receptors are present as ionotropic $\left(\mathrm{GABA}_{\mathrm{A}}\right.$ receptor) and metabotropic $\left(\mathrm{GABA}_{\mathrm{B}}\right.$ receptor) postsynaptic receptors that are bound by GABA, the major 
inhibitory neurotransmitter in the central nervous system (CNS). While long-range GABAergic neurons do exist, the majority of research attention relevant to cognition has focused on GABAergic interneurons, which appear to have a central role in the synchronisation of network activity and the generation of oscillations in different frequency bands, processes felt to facilitate the efficacy of information processing ${ }^{1}$.

Furthermore, the inhibitory-excitatory balance that is emergent from dynamic interactions of glutamatergic and GABAergic signalling is thought to play an important role in stimulus representation and information propagation and therefore is likely to be crucial not only for cognition but for behavioural processes defined more broadly ${ }^{2,3}$.

\section{Autoimmune encephalitis}

Detailed neuropsychological characterisation is often challenging in the acute phase of autoimmune encephalitis due to the severity of clinical symptoms. Accordingly, in the acute phase clinical descriptions tend to be qualitative and the more extensive cognitive testing possible in the post-acute and chronic phases is frequently authored from a neurorehabilitation perspective, potentially introducing a selection bias towards cases with more severe dysfunction. While we describe the acute and chronic cognitive deficits separately, in practice such distinctions are not so easily delineated and there is often significant overlap. Table 1 summarises the cognitive impairment associated with each neuronal autoantibody-associated encephalitis. It is useful to note at the outset that while most autoimmune encephalitides are named after the putatively pathogenic antibody, there is increasing evidence that there may be variability in the breadth of the antibody response between these disorders; for example, in LGI1 encephalitis the polyclonal antibody response appears only to target the LGI1 protein ${ }^{4}$, whereas in NMDAR encephalitis less than $10 \%$ of intrathecal antibody-secreting cells are specific for the NR1 subunit of the NMDAR ${ }^{5}$. This raises the possibility that antibodies targeting other (non-canonical) epitopes, or even entirely different proteins, may contribute to the clinical expression of disease in some disorders.

\section{NMDAR encephalitis Acute phase}

NMDAR encephalitis is both the most common and best-defined cause of autoimmune encephalitis. Its onset is often heralded by an influenza-like prodrome followed by a characteristic progression from psychotic symptoms and cognitive impairment to seizures, movement disorder, autonomic instability and loss of consciousness ${ }^{6}$. While most frequently described in women of child-bearing age, it is increasingly recognised in children and older adults of both sexes ${ }^{6}$.
Cognitive dysfunction is often profound and in the acute phase typically extends across all domains. Deficits in executive function and memory are most marked but attention, language, visuospatial processing and social cognition are also affected to varying degrees ${ }^{7,8}$. Atypical, unusual presentations of cognitive impairment are occasionally described in NMDAR encephalitis; case reports illustrate disruption to temporal orientation with a loss of age awareness and also transient epileptic amnesia, characterised by repeated, brief episodes of anterograde and retrograde amnesia ${ }^{9,10}$. Another recent case report documented a patient who, due to the nature of their presentation with memory loss, cognitive fluctuations, visual hallucinations and sleep disorder, was initially misdiagnosed with Lewy Body Dementia before NMDAR antibodies were identified leading to effective immunotherapy treatment ${ }^{11}$. In older adults with NMDAR encephalitis cognitive impairment is often more prominent which may account for some of the variability seen ${ }^{12}$.

\section{Long term follow-up}

While cognitive dysfunction is extensive in the acute phase it can also persist for years after the initial insult ${ }^{13}$. As in the acute phase, episodic memory and executive function are most consistently affected 1 year after initial presentation $^{7,8,13,14}$. A recent systematic review found chronic cognitive impairment in up to three quarters of patients, with timely immunotherapy the most important factor determining positive outcomes ${ }^{8}$. This emphasises the persistent and major morbidity of cognitive impairment in anti-NMDAR encephalitis for patients, in addition to the importance of early diagnosis and appropriate treatment ${ }^{13}$.

\section{Mechanisms underlying cognitive impairment}

The NMDA receptor is a tetrameric ligand-gated ion channel which mediates excitatory transmission in the CNS and is crucial for LTP, the neural substrate for learning and memory. In NMDAR encephalitis, there is substantial intrathecal production of IgG NMDAR antibodies which target the NR1 subunit of the receptor, causing a reversible and titre-dependent internalisation of the NMDA receptor with subsequent reduced receptor density and reduction in NMDAR-mediated currents ${ }^{15,16}$. The antibody-mediated disruption of the interaction between the NMDA and ephrin-B2 receptor is central to this, causing the NMDAR to become displaced which allows subsequent internalisation ${ }^{17}$. Indeed, animal studies suggest that if ephrin-B2 is co-administered, the pathogenic effects of the antibodies are blocked and no downstream effects are seen ${ }^{18}$.

Accordingly, NMDAR-dependent LTP is depressed in mouse hippocampal slices with prolonged exposure to CSF from patients with NMDAR encephalitis ${ }^{19}$. 


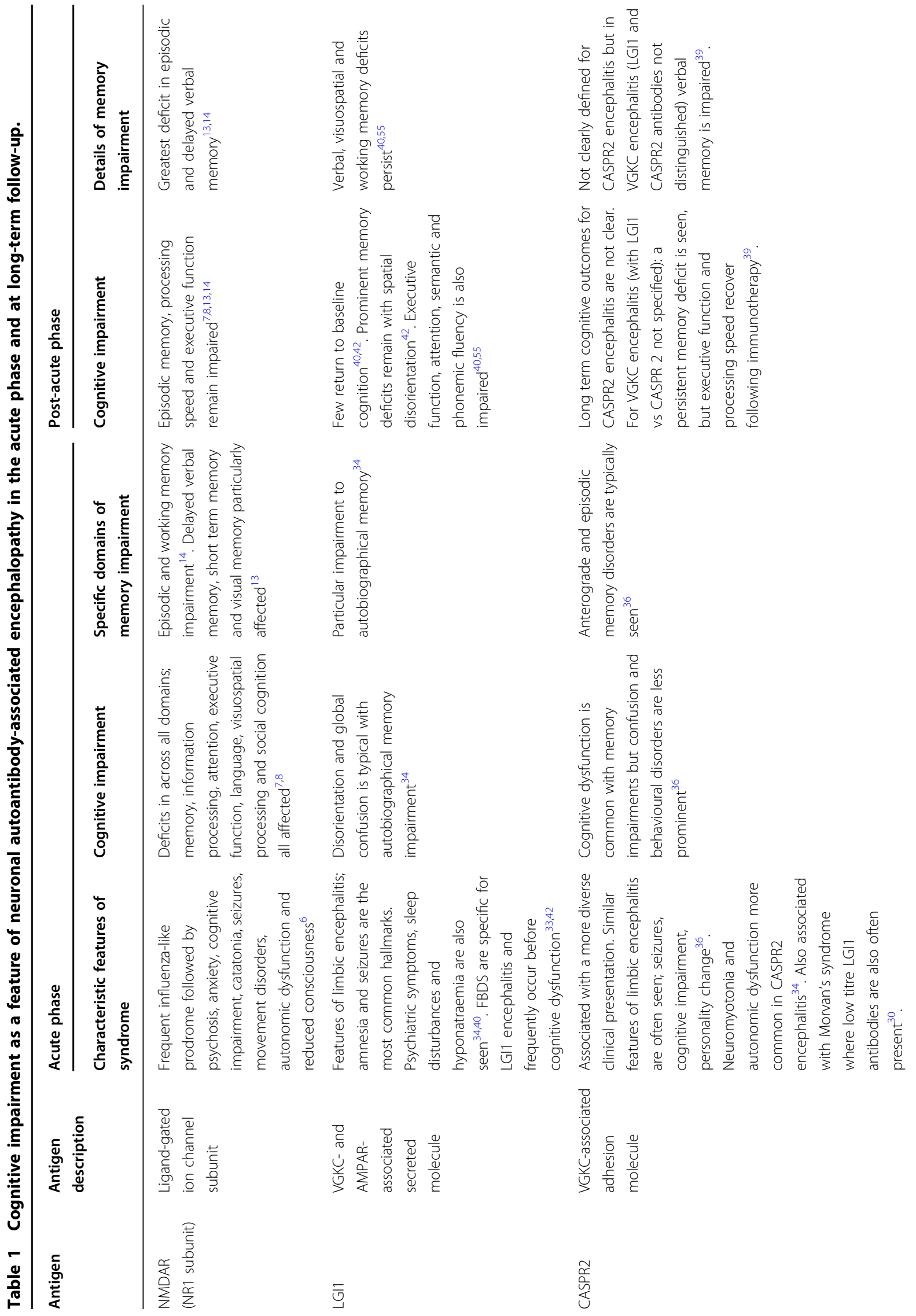

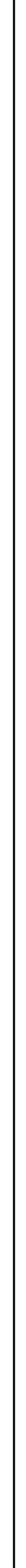




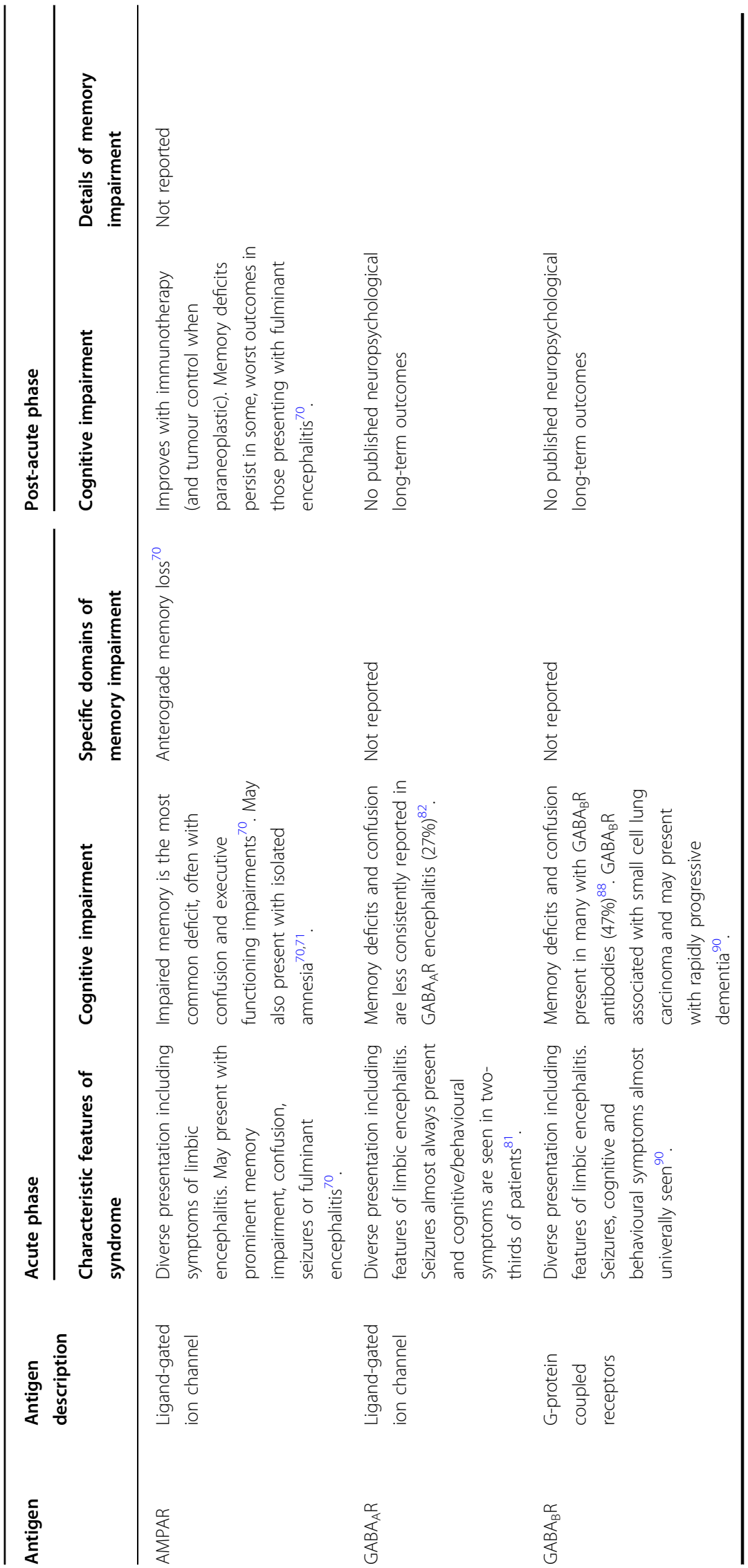


Furthermore, in vivo infusion of NMDAR antibodies reduces excitatory postsynaptic currents in rat hippocampal neurons and simultaneously impairs learning and spatial working memory ${ }^{20}$. Infusion of inflammatory cytokines also reduced excitatory postsynaptic currents and led to further impairment in learning performance suggesting there may be other additive factors influencing cognitive dysfunction ${ }^{15}$. More recently, object recognition has been shown to be impaired following injection of NMDAR antibodies to rat hippocampi, widening the application of NMDAR antibody-mediated cognitive dysfunction $^{21}$. NMDAR antibodies cause a dose-dependent increase in extracellular glutamate, akin to the elevated level of glutamate following ketamine administration which is associated with cognitive effects ${ }^{22}$.

NMDARs are highly concentrated in the hippocampus and frontal cortex which likely underlies the predominance of cognitive deficits in episodic memory and executive function ${ }^{23}$. Indeed, reduced functional connectivity between the hippocampus and the medial prefrontal cortex and impaired connectivity within the medial temporal lobe (MTL) network was observed in patients with NMDAR encephalitis and shown to predict the severity of memory impairment (Fig. 1) ${ }^{24,25}$. Furthermore, although the disorder is not a classical limbic encephalitis, in the post-acute phase reduced bilateral hippocampal volume and microstructural integrity is observed, which likewise correlates with memory impairment ${ }^{26}$. Widespread damage to superficial white matter - which encompasses short-range association fibres and intracortical myelin-additionally contributes to impairments of attention and memory ${ }^{27}$, and extensive changes in deep white matter integrity correlate with disease severity ${ }^{24}$. This structural damage to hippocampus and white matter suggests pathological mechanisms ongoing beyond the demonstrated reversible internalisation of NMDAR without damage to neurons; ${ }^{16,26} \mathrm{~T}$ cell mediated processes may have an as-yet under-appreciated role here ${ }^{28}$.

\section{Encephalitis formerly attributed to antibodies to the voltage gated potassium channel (VGKC): LGI1 and CASPR2 encephalitis}

The first potentially reversible, immunosuppressionresponsive form of limbic encephalitis was described nearly 20 years ago $^{29}$. The VGKC antibodies originally detected were believed to directly target the Kv1.1, 1.2 and 1.6 channels. However, it is now understood that pathological VGKC antibodies target the extracellular domains of one or more of three proteins tightly complexed with VGKCs; LGI1, CASPR2 and contactin-2, each with different implications ${ }^{30}$. "Double negative" VGKC antibodies - that is VGKC antibody positivity without LGI1 or CASPR2 positivity-are of questionable clinical significance (potentially targeting intracellular targets) and for this reason VGKC antibodies should not be routinely tested in the initial investigation of a patient with a suspected autoimmune CNS disorder ${ }^{31,32}$.

\section{LGI1 encephalitis \\ Acute phase}

Typical presenting symptoms of LGI1 encephalitis are those of a limbic encephalitis; symptoms include cognitive impairment, behavioural changes and focal seizures. Seizures typically precede the onset of cognitive impairment, with a progressive amnesia usually developing at their crescendo $^{33}$. Unlike other autoimmune encephalitides (and most autoimmune conditions) LGI1 encephalitis is most common in middle-aged males. Although LGI1 encephalitis is phenotypically similar to other paraneoplastic and non-paraneoplastic limbic encephalitides, faciobrachial dystonic seizures (FBDS) are unique to LGI1 encephalitis and are a useful clinical differentiator ${ }^{34}$.

Prominent amnesia is a hallmark of limbic encephalitis associated with LGI1-antibodies; autobiographical memory is particularly impaired, often with significant confusion and disorientation ${ }^{30,35,36}$. Isolated amnestic syndrome can occur in up to $10 \%$ of cases of LGI1 encephalitis ${ }^{35}$ and, in the absence of seizures and where the onset is insidious, LGI1 encephalitis can mimic other syndromes of cognitive impairment such as neurodegenerative dementias ${ }^{37}$. Indeed, numerous case reports have documented LGI1 encephalitis misdiagnosed as Alzheimer's disease, Creutzfeld-Jacob Disease and Dementia with Lewy Bodies before further investigation elucidated the true cause and led to reversal of the cognitive impairment with immunotherapy ${ }^{38}$.

\section{Long term follow-up}

Looking at long term outcomes for patients with previously defined VGKC antibodies collectively, without differentiating LGI1 or CASPR2-positive patients, cognitive deficits correlate with antibody titre and are most marked for verbal memory, while processing speed and executive function are relatively spared ${ }^{39}$. In LGI1 encephalitis, most patients have a chronic cognitive impairment, with memory predominantly affected but deficits of attention and executive function also reported ${ }^{40-42}$. Greater disease severity, delays to immunotherapy or longer courses of immunotherapy (likely mandated by greater disease severity) are all associated with more profound cognitive dysfunction in LGI1 encephalitis ${ }^{40}$. Indeed, early treatment of isolated FBDS significantly reduces the risk of developing cognitive impairment, highlighting the importance of recognising FBDS early ${ }^{43,44}$.

\section{Mechanisms underlying cognitive impairment}

Unlike NMDAR antibodies, LGI1 antibodies are predominantly of the IgG4 subclass, although the IgG1 subclass may also contribute to pathology. LGI1 is a 


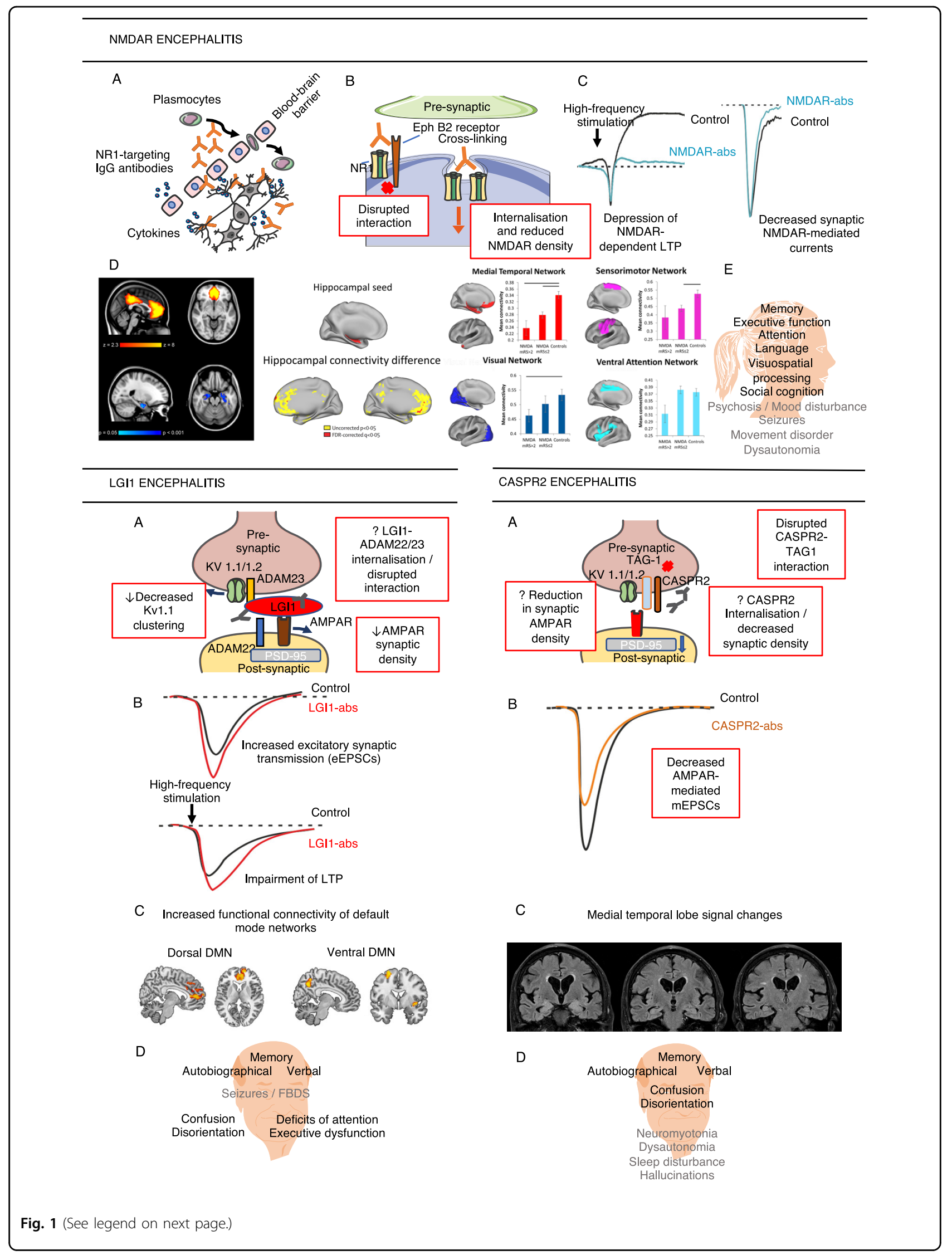


(see figure on previous page)

Fig. 1 NMDAR encephalitis. a NMDAR-antibody access to the brain; b Molecular mechanisms of pathogenicity; $\mathbf{c}$ Functional effects of the NMDARantibodies; d Functional connectivity changes in NMDAR encephalitis. (Left) Impaired functional connectivity between the hippocampus and the medial prefrontal cortex identified using independent component analysis and dual regression. The severity of hippocampal functional connectivity impairment correlates with individual memory deficits. (Middle) Impaired connectivity of the hippocampus with the medial prefrontal cortex replicated using a seed-based approach. In addition, reduced hippocampal connectivity with other regions of the default mode network was observed, e.g. the posterior cingulate cortex and the precuneus. (Right) Using a network-based approach, significantly reduced functional connectivity was found within several large-scale networks, including the medial temporal lobe network, the sensorimotor network and the visual network. [Figures reproduced with permission from ${ }^{24,25}$ ] e Clinical phenotype. LGI1 encephalitis: a Molecular mechanisms of pathogenicity; b Functional effects of the LGI1-antibodies; c Patients with LGl1 encephalitis had increased functional connectivity of the dorsal and ventral default mode network (DMN) that correlated with working memory (dorsal DMN) and episodic memory (ventral DMN) performance. [Figures reproduced with permission from ${ }^{55}$; d Clinical phenotype. CASPR2 encephalitis and Morvan's Syndrome: a Molecular mechanisms of pathogenicity; b Functional effects of the CASPR2-antibodies; c Bilateral FLAIR hyperintense signal and mild atrophy of the hippocampus in a patient with CASPR2 encephalitis; $\mathbf{d}$ Clinical phenotype. Components of this figure were created using Servier Medical Art templates, which are licensed under a Creative Commons Attribution 3.0 Unported License; https://smart.servier.com. The shapes of the electrophysiological traces were modelled on data published $\mathrm{in}^{47,61,147}$.

trans-synaptic protein which complexes presynaptic ADAM23 with postsynaptic ADAM22. LGI1 antibodies act directly to disrupt this binding which subsequently reduces synaptic AMPAR density ${ }^{45}$. However, given the marked differences in clinical presentation with AMPAR encephalitis, it is likely that LGI1 antibodies have other downstream effects in addition to modulation of AMPARs. In one study of 103 patients, IgG1 antibodies occurred more frequently in patients with cognitive impairment, suggesting a role for complement-mediated pathology in this symptom domain ${ }^{43}$. Serum IgG VGKC antibodies from patients with limbic encephalitis led to cell excitability with an increased tonic rate of firing and strengthened mossy fibre-evoked synaptic responses in CA3 rat hippocampal slices-the sera used in this study was later found to have LGI1 rather than CASPR2 antibodies $^{46}$. A selective VGKC antagonist mimicked these effects suggesting that the antibody-mediated increase in cell excitability is directly related to reduction in VGKC function. Petit-Pedrol et al. later demonstrated LGI1 antibody-mediated reductions in synaptic density of both $\mathrm{K}_{\mathrm{v}} 1.1$ VGKC and AMPAR receptors with simultaneous, reversible memory deficits ${ }^{47}$. They observed hyperexcitability, increased glutamatergic transmission and reduced synaptic failure rate with a severe impairment to neuronal plasticity and LTP in CA1 and the dentate gyrus. A recent study further explored the pathogenic mechanisms of LGI1-antibodies and suggested the possibility of synergistic contributions. Monoclonal antibodies derived from patients with LGI1-mediated disease were found to target multiple epitopes, with distinct specificities to the protein's LRR and EPTP-domains. In this study, LRR and EPTP-binding LGI1 antibodies seemed to mediate distinct functional effects. LRR-directed antibodies bound and internalised the LGI1-ADAM22/23 complexes. Surprisingly, this mechanism was independent of IgG bivalency, as both IgG4 antibodies and Fab fragments retained this capacity. The dominant effect of EPTP-binding antibodies, on the other hand, appeared to be disruption of the interaction between LGI1 and its receptors. Both LRR- and EPTP-specific antibodies completely abrogated LTP induction at CA3-CA1 hippocampal synapses, which translated to impairment of recognition memory in mice exposed to LRRP-antibodies ${ }^{48}$. These findings likely explain the amnestic syndrome experienced by patients with LGI1 encephalitis.

Interestingly, in humans, LGI1 gene mutations lead to autosomal dominant lateral temporal lobe epilepsy characterised by frequent partial seizures often with auditory auras (rather than FBDS) but typically without cognitive impairment ${ }^{49}$. This might relate to differences in the timing of protein dysfunction (from early neurodevelopment vs later in adult life) or to other in vivo roles of LGI1 and its targeting by autoantibodies that have not yet been clearly defined.

In the rodent, LGI1 and CASPR2 are preferentially expressed in CA3 and CA1 hippocampal subfields ${ }^{30,50}$. Correspondingly, MR imaging in the acute phase of LGI1 or CASPR2 encephalitis is usually abnormal, typically showing inflammation in MTL, an area critically involved in memory processing ${ }^{51,52}$. At follow-up, almost all patients show some degree of hippocampal atrophy, which is often bilateral, with loss seen particularly in the CA3 subfields ${ }^{40,53}$. The volumetric atrophy also correlates with the severity of episodic autobiographical and verbal memory impairments ${ }^{40,53}$. The microstructural integrity of the hippocampus is also impaired on a wider level which associates with both disease severity and memory function; functional connectivity of the remaining hippocampus correlates closely with the degree of memory impairment $^{40,54}$. Interestingly, functional connectivity analyses also show characteristic alterations in several large-scale networks, suggesting that LGI1 encephalitis is not confined to the limbic system. Increased connectivity in the ventral and dorsal default-mode network is associated with improved memory performance, indicating a 
compensatory mechanism, while connectivity in the salience network is reduced and correlated with impaired memory function ${ }^{55}$. These network changes indicate cognitive deficits beyond mere memory impairment in LGI1 encephalitis, suggesting brain-wide alteration of the connectome triggered by focal hippocampal damage.

\section{CASPR2 encephalitis and Morvan's syndrome Acute phase}

CASPR2 antibodies associate with a wide range of neurological syndromes, which often overlap in the same patient. Manifestations include peripheral nerve hyperexcitability (often referred as neuromyotonia), neuropathic pain, paroxysmal movement disorders and limbic encephalitis ${ }^{56}$. Among the different CASPR2-related syndromes, limbic encephalitis and Morvan's syndrome have a particular impact on cognitive functions. CASPR2 encephalitis, similarly to LGI1 encephalitis ${ }^{57}$, is characterised by limbic dysfunction, with temporal seizures, memory impairment and frontal dysfunction ${ }^{36}$. Morvan's syndrome, a rarer disorder, is characterised by peripheral nerve hyperexcitability and encephalopathy, in addition to sleep disturbance, hallucinations, dysautonomia and pain. Whether Morvan's syndrome is a distinct entity or merely a combination of autoimmune encephalitis and peripheral nerve hyperexcitability is unclear, but some suggest there are sufficient differences to consider it a distinct syndrome. Limbic dysfunction, such as temporal seizures, anterograde amnesia or hyperintensities in the MRI are uncommon in Morvan's syndrome, except in patients who are both CASPR2 and LGI1-antibody positive. Both CASPR2 limbic encephalitis and Morvan's syndrome are more common in elderly male patients, but association with thymoma and other autoimmune diseases is much more common in Morvan's syndrome.

\section{Long term follow up}

Data on long term outcomes, particularly on cognitive sequelae, are remarkably scarce in CASPR2 encephalitis and Morvan's syndrome, and it is often pooled together with that of LGI1 encephalitis or under the previous umbrella designation "VGKC encephalitis". Partial or full recovery after immunosuppression is usually the norm in non-paraneoplastic CASPR2 encephalitis or Morvan's syndrome $^{36,57}$. However, relapses are frequent, particularly in the form of increased seizure activity, and are usually steroid-responsive.

\section{Mechanisms underlying cognitive impairment}

CASPR2, a member of the neurexin family, is a cell adhesion transmembrane protein first identified in the VGKC clusters (mainly Kv1.1 and Kv1.2) at the juxtaparanodes of myelinated neurons ${ }^{59}$. CASPR2 stabilises the VGKCs such that antibody-mediated disruption of this protein causes peripheral hyperexcitability syndromes. The role of CASPR2 at the CNS synapse, however, is not well known, but the bulk of data suggests important functions in synaptic processes and neuronal activity. CASPR2 was implicated in the trafficking of AMPA receptors to the synaptic membrane ${ }^{60,61}$, suggesting that glutamatergic transmission dysfunction could underpin the cognitive impairment seen in CASPR2-mediated CNS disease. Others suggest that CASPR2 has a role in inhibitory hippocampal synapses, and that the antibodymediated perturbation of inhibitory interneuron activity could lead to increased neuronal hyperexcitability and ultimately to the seizures suffered by these patients ${ }^{62,63}$.

Like LGI1-antibodies, CASPR2-antibodies are predominantly of the IgG4 subclass, although IgG1 antibodies can also be present and are potential contributors to pathology. They too target multiple epitopes, with the protein's N-terminal discoidin-like and laminin G1 domains being obligatory epitopes ${ }^{36,64}$. The mechanisms by which these antibodies cause disease, however, are still not completely understood and there have been conflicting results in the literature. While some report absence of CASPR2 internalisation ${ }^{63,65}$, others suggest that CASPR2 is indeed internalised ${ }^{35,61,66}$ by the antibodies. It is possible that the IgG subclass (IgG1 vs IgG4) titre differences in the CASPR2-IgG preparations used in these studies, as well as differences in the in vitro systems used, partially account for these contradicting results. Those groups that found no evidence for antibodymediated internalisation suggest that CASPR2-antibodies exert their function through interference with the interaction between CASPR2 and TAG- ${ }^{63,65}$. Finally, CASPR2-antibodies may also exert their pathogenicity by altering the protein's known function in AMPAR synaptic traffic. A recent study showed significant synaptic loss of AMPARs in cortical neurons incubated with IgG1 and IgG4 CASPR2 antibodies, while in vivo injection of the same antibodies in the mouse visual cortex significantly decreased AMPAR-mediated currents ${ }^{67}$.

These results suggest that CASPR2 likely has different functions in different synapses, which would imply different but synergistic effects of the CASPR2-antibodies in the pathophysiology of encephalitis or Morvan's syndrome. More studies on the pathogenic mechanisms of CASPR2-antibodies are necessary.

\section{Other neuronal autoantibody-mediated encephalitis AMPAR encephalitis \\ Acute phase}

Due to its rarity, the clinical course of AMPAR encephalitis is not yet well characterised. Most studies describe limbic dysfunction at onset characterised by anterograde and retrograde amnesia, confusion, psychiatric symptoms 
and seizures ${ }^{68}$. First described in a series of ten patients, it was reported to mostly affect older women, often with an underlying malignancy and high rates of relapse ${ }^{69}$. The phenotype has since widened with marked heterogeneity in presentation observed ${ }^{68,70}$. However, cognitive dysfunction remains a universally prominent feature and isolated amnesic syndromes have also been observed with a focal impairment to anterograde memory ${ }^{70,71}$. Indeed, in a review of 18 cases there was evidence of cognitive impairment in all, ranging from anterograde memory impairments and executive dysfunction to generalised confusion $^{68}$. A recent systematic review identified 55 patients with AMPAR encephalitis; a diverse phenotype was observed but amnesia was recognised as the most common clinical symptom ${ }^{71}$. However, amnesia at onset was also associated with greater diagnostic delays, highlighting the need for improved recognition of the symptomatic profile associated with AMPAR encephalitis.

\section{Long term follow-up}

There are limited data available on the neuropsychological outcomes of AMPAR encephalitis patients. Case reports have described considerable neurocognitive improvement at follow-up with a third achieving complete recovery, but this has yet to be characterised quantitatively ${ }^{72}$. Although in general outcomes appear favourable, psychiatric symptoms or fulminant encephalopathy at onset is associated with poor prognosis at follow $\mathrm{up}^{70,72}$. In a case report, significant memory impairment was found to persist 1 year after disease onset, accompanied by hippocampal atrophy, persistent hippocampal hypermetabolism in ${ }^{18} \mathrm{FDG}$ PET imaging and ongoing epileptic activity on $\mathrm{EEG}^{73}$.

\section{Mechanisms underlying cognitive impairment}

AMPARs are glutamate-gated ion channels composed of combinations of the tetrameric subunits GluA1-4. AMPARs mediate much of the rapid, excitatory neurotransmission in the brain and are integral to $\mathrm{LTP}^{74}$. The composition of subunits has important consequences for the role of AMPAR in synaptic plasticity and typically AMPAR antibodies target GluA1 and GluA2 subunits ${ }^{75}$. AMPAR antibodies cause reductions in AMPAR expression with changes to their synaptic localisation through receptor internalisation and degradation ${ }^{69,76}$. Reductions in AMPAR-mediated currents are seen with alterations in the patterns of action potential firing and an increase in intrinsic excitability of neurons likely due to a compensatory decrease in inhibitory synaptic transmission ${ }^{75,76}$. Haselmann et al. demonstrated antibody-mediated internalisation of GluA2-containing AMPARs with compensatory insertion of mostly GluA1-containing AMPARs ${ }^{77}$. The subsequent LTP impairments were hypothesised to be secondary to the reduced availability of extrasynaptic
AMPAR, on which LTP is dependent ${ }^{77,78}$. Alongside the LTP changes, they found in vivo impairments to learning and memory; this was the first animal model to recapitulate the severe memory impairments typical of AMPAR encephalitis ${ }^{77}$.

Although ubiquitous, GluA1/2 and GluA2/3 are particularly expressed in the hippocampal and limbic regions, and as such these regions are particular targets for AMPAR antibodies ${ }^{69}$. Indeed, in the vast majority brain MRI is abnormal in the acute phase, often showing bilateral temporal lobe enhancement, reflecting areas of greatest AMPAR density ${ }^{71}$. Given AMPARs are found throughout the brain, albeit at lower concentrations than in the limbic regions, autoantibody binding in other regions could account for the marked heterogeneity seen in clinical profile, with the more generalised distribution also underpinning the global atrophy and hypometabolism reported in some cases of AMPAR encephalitis ${ }^{79}$.

\section{$\mathbf{G A B A}_{\mathbf{A}} \mathbf{R}$ encephalitis}

$\mathrm{GABA}_{\mathrm{A}} \mathrm{R}$ antibody-mediated encephalitis has a broad clinical phenotype affecting all ages of both sexes ${ }^{80,81}$. The largest case series to date confirmed seizures as the most frequent symptom with altered cognition evident in twothirds of patients ${ }^{81}$. While the clinical phenotype has yet to be fully characterised, greater variability in presentation is evident with memory deficits not ubiquitous and cases without seizures also described ${ }^{82,83}$. It is possible that the variation in clinical presentation of $\mathrm{GABA}_{\mathrm{A}} \mathrm{R}$ antibody encephalitis across these series may be due to the differences in subunit specificity of cell-based assays used to detect the antibodies. Studies using the $\alpha 1$ and $\beta 3$ subunits $^{80,81,84}$ have tended to find a more restricted phenotype than those using the $\alpha 1, \beta 2$ and $\gamma 2$ subunits $^{82}$, although differences may also reflect the different nature of the patient populations whose samples were tested in these studies.

\section{Mechanisms underlying cognitive impairment}

GABA receptors are the major mediator of inhibitory synaptic transmission in the $\mathrm{CNS} . \mathrm{GABA}_{\mathrm{A}}$ receptors are ligandgated chloride ion channels, underpinning fast synaptic inhibition, while $\mathrm{GABA}_{\mathrm{B}}$ receptors are G-protein coupled receptors modulating slower inhibitory transmission. Autoantibodies to $\mathrm{GABA}_{\mathrm{A}}$ Rs are generally IgG1 and those which target the extracellular epitope of the $\gamma 2$, $\alpha 1$ and $\beta 3$ subunit cause reduced synaptic and extrasynaptic $\mathrm{GABA}_{\mathrm{A}} \mathrm{R}$ with consequent reductions in inhibitory postsynaptic currents in vitro ${ }^{80,85}$. Mutations to the $\mathrm{GABA}_{\mathrm{A}} \mathrm{R}$ reducing expression levels cause generalised epilepsies but there are, as yet, no animal studies which demonstrate the impact of $\mathrm{GABA}_{\mathrm{A}} \mathrm{R}$ antibodies in vivo ${ }^{86}$. However, in the acute phase brain MR imaging is commonly abnormal; $77 \%$ show multifocal, asynchronous grey 
and white matter changes most often in the temporal and frontal lobes ${ }^{81}$. These widespread changes reflect the extensive distribution of $\mathrm{GABA}_{\mathrm{A}} \mathrm{R}$ which, along with the likely presence of additional anti-neuronal antibodies, may underpin the heterogeneity in presentation of $\mathrm{GABA}_{\mathrm{A}} \mathrm{R}$ encephalitis ${ }^{87}$.

\section{$G A B A_{B} R$ encephalitis}

$\mathrm{GABA}_{B} \mathrm{R}$ encephalitis was first described in a case series of 15 patients characterised by seizures and memory deficits $^{88}$. Older adults are most affected and there is a strong association with small cell lung cancer, occurring in up to $50 \%$ of patients and associated with poorer outcomes $^{89}$. While more recent clinical descriptions have expanded the clinical phenotype, cognitive impairment and seizures remain the central symptoms, almost universally affecting patients in the acute phase but the nature of neuropsychological impairments have not been examined in detail ${ }^{90}$. Interestingly, this recent case series identified a subset of patients with $G_{A B A} R$ encephalitis presenting with a "rapidly progressive dementia" with subacute cognitive impairment in the absence of seizures $^{90}$. Prognosis is often poor, with a median survival of 17 months, and the long term outcomes for $G_{A B A} R$ encephalitis have yet to be studied ${ }^{90}$.

\section{Mechanisms underlying cognitive impairment}

Antibodies associated with $\mathrm{GABA}_{\mathrm{B}} \mathrm{R}$ encephalitis are predominantly of the IgG1 subclass targeting the extracellular domain of the $\mathrm{B} 1$ subunit $^{88}$. Autoantibodies to the $\mathrm{GABA}_{\mathrm{B}} \mathrm{R}$ act to inhibit channel function rather than internalise or deplete cell surface receptor levels ${ }^{91}$. In line with the clinical phenotype, knockout GABAB1R mice exhibit spontaneous seizures with marked memory impairment ${ }^{92}$. The $\mathrm{GABA}_{\mathrm{B}}$ receptor is mainly expressed in hippocampus, amygdala, thalamus, and cerebellum reflecting the common MTL abnormalities seen in imaging during the acute phase of encephalitis ${ }^{93}$.

\section{Beyond autoimmune encephalitis}

While the impact of neuronal autoantibodies on cognition is well established within each encephalitic syndrome, their role outside this context is less clear. However, there is accumulating evidence to indicate these antibodies (particularly NMDAR antibodies) may be of relevance outside clinically defined encephalitis, in patients without evidence of frank encephalopathy. All NMDAR antibodies, irrespective of immunoglobulin class and donor source, demonstrate pathological potential; in vitro (and, to a lesser extent, in vivo) instigating NMDAR internalisation and dysfunctional glutamatergic signalling ${ }^{94}$. However, this is not equivalent to asserting that all such antibodies are potentially encephalitogenic and this distinction must be held in mind ${ }^{95}$.
Serum NMDAR antibodies, of uncertain clinical relevance, are found in appreciable numbers in healthy controls, which is a challenge to the view that they are universally pathological. In one such study serum NMDAR antibodies of all isotypes occurred at an overall frequency of about $10 \%$, increasing with age but not differing according to disease status; NMDAR IgG remained relatively rare however, detectable in around $1 \%$. Similar results were reported for antibodies to other antigens, although these were much less common than NMDAR antibodies ${ }^{96}$. Notably, different assays also appear to have different sensitivities for detection of neuronal autoantibodies. Live cell-based assays, in which sera or CSF is applied to recombinant HEK cells expressing the antigen of interest before fixation, appear to detect many more positive specimens than do fixed assays, in which the sera or CSF is applied after fixation. Nonetheless, while live CBAs do detect antibodies that demonstrably bind their target, the clinical relevance of the results is less clearthat is, the increased analytical sensitivity of these assays may come at the expense of clinical specificity ${ }^{97,98}$.

In part because of the issue of non-specificity of serum autoantibodies to neuronal antigens, diagnostic criteria for autoimmune CNS disorders place much emphasis on paraclinical investigations which are required to determine the clinical relevance of a positive antibody, such as MRI or EEG. Neither in consensus criteria for autoimmune encephalitis ${ }^{99}$ or for autoimmune psychosis ${ }^{100}$ can a positive serum antibody on its own lead to a diagnosis of probable antibody-mediated disorder.

It has been suggested that some aspect of blood-brain barrier permeability is one factor which can determine the clinical relevance of a positive serum antibody, with numerous studies showing autoantibody-mediated neuropsychiatric symptoms to be dependent on blood-brain barrier permeability ${ }^{101-103}$. Indeed, in SLE rodent models, infusion of NMDAR antibodies (targeting the NR2 rather than NR1 subunit) only caused cognitive impairment where the blood-brain barrier was disrupted ${ }^{104}$. It also remains possible that impaired blood-brain barrier integrity, or other mechanisms, could allow formation of antibodies in patients with neuronal decline via recognition of these neuronal antigens for the first time ${ }^{105}$. We review the evidence for a role of autoantibody-associated cognitive impairment in various common disorders.

\section{Cancer}

Paraneoplastic neurological syndromes are immunemediated disorders triggered by tumours driving the immunization process. Many of the autoimmune encephalitis-associated antigens are expressed by tumours, and paraneoplastic neurological syndromes can be associated with neuronal autoantibodies targeting these tumour-expressed neuronal antigens (such as 
NMDAR antibodies with teratomas and CASPR2 antibodies with thymomas). However, the association between tumours, neuronal antibodies and cognition has recently been explored beyond the context of limbic encephalitis. In a retrospective study, neuronal antibodies were observed in almost a quarter of cancer patients tested and cognitive deficits were found to be significantly more common in those with a positive serum neuronal antibody ${ }^{106}$. The antibodies most commonly identified were IgA or IgM NMDAR antibody, and the level of cognitive impairment was related to the degree of bloodCSF barrier disruption as indexed by the cerebrospinal fluid/serum albumin quotient ${ }^{106}$. These findings were then replicated and extended in a prospective study of melanoma patients ${ }^{107}$. Importantly, all patients underwent a comprehensive cognitive assessment that was performed blinded to antibody status. Melanoma patients with neuronal autoantibodies (mostly serum IgA and IgM NMDAR antibodies) showed more than threefold higher odds for cognitive impairment than melanoma patients without antibodies. Furthermore, the degree of cognitive impairment was correlated with the titre of NMDAR IgM or IgA antibody ${ }^{107}$. Affected cognitive domains included memory, attention and executive function indicating neuronal autoantibodies may have a role as both a pathophysiological factor and a potential biomarker for cognitive impairment ${ }^{107}$. However, future studies are needed to determine whether the observed cognitive impairments in antibody-positive patients are specific to cancer and whether antibodies themselves are pathogenic or rather indicate pathophysiological states leading to cognitive decline.

\section{Viral encephalitis}

It is now established that neuronal autoimmunityprincipally to NMDAR but other antigens have also been implicated - can be initiated by herpes simplex encephalitis (HSE) ${ }^{108,109}$. In up to $90 \%$ of so-called 'relapses' of HSE, where the clinical picture is frequently dominated by cognitive dysfunction, the aetiology is now understood to represent a "secondary autoimmune encephalitis" responsive to immunotherapy; indeed autoimmune encephalitis is thought to occur in around a third of HSE patients $^{58}$. One obvious factor potentially responsible for initiation of autoimmunity is the gross neuronal destruction and subsequent epitope exposure caused by HSV infection. Indeed, other CNS viral infections are also known to initiate neuronal autoantibody production ${ }^{109}$. However, history of non-encephalitic HSV infection is also more common in NMDAR encephalitis ${ }^{110}$ suggesting molecular mimicry may also play a role ${ }^{111}$. Interestingly in one study, even in patients who did not develop frank encephalopathy after HSV infection, CSF NMDAR antibodies were interpreted to be predictive of the degree of improvement in cognitive function in the recovery phase of $\mathrm{HSE}^{112}$. However, in this study, there was no difference in cognitive performance between NMDAR antibody positive and negative patients at any time during followup; rather, there was only a significantly greater improvement of cognitive scores in the antibody negative group, driven by recovery from a worse baseline performance for these patients compared to that of the NMDAR antibody positive patients. Moreover, the relatively impaired performance of NMDAR antibody negative patients at baseline was driven by four outliers with particularly impaired performance ${ }^{112}$. Given the significant impact of post-HSE cognitive impairment on functioning and quality of life, and the lack of clarity of these results, attempts at clarifying the possible prognostic significance of NMDAR antibodies in this patient group are of continued interest. Overall it appears that secondary neuronal autoimmunity following other kinds of brain tissue damage could have a role in shaping the extent of cognitive dysfunction following an acute event. One such obvious example of brain tissue damage, amenable to study by virtue of its frequency, is stroke.

\section{Stroke}

Serum neuronal antibodies are detected in up to onefifth of patients following acute stroke ${ }^{113}$. However, there is as yet no consensus regarding the relevance of these antibodies in this clinical population. One recent study did not find any association of serum neuronal antibody seropositivity with functional outcome or clinical features in acute stroke $\mathrm{e}^{114}$. Another large study did not find an association with seroprevalence per se, unless the group was stratified by NMDAR antibody titre, when high antibody titre was found to correlate with poor functional outcomes ${ }^{115}$. In addition, NMDAR antibody seropositive patients had an increased risk of secondary vascular events or death. The integrity of the blood-brain barrier may also be relevant in stroke: in patients with acute ischaemic stroke, NMDAR antibodies were associated with larger stroke lesions in patients with a "leaky" bloodbrain barrier, as indicated by APOE4 status, and conversely, in patients with an intact blood-brain barrier NMDAR antibodies were associated with smaller stroke lesion size $\mathrm{s}^{113}$. Thus, the relationship between autoantibodies and outcomes following stroke is not linear but serum NMDAR antibodies may be of particular relevance where they are found at high titre and/or with a compromised blood-brain barrier. Although functional outcomes are in part driven by cognitive status following stroke, to date no study has investigated an association between antibody seropositivity and quantitative cognitive outcomes following stroke. This would be methodologically challenging due to the inherent variability in cognitive outcomes with lesion heterogeneity but could offer 
the potential for new insights into the impact of neuronal antibodies outside encephalitis.

\section{Psychiatric disorders}

Psychiatric symptoms are hallmarks of many of the autoimmune encephalitic syndromes, in some cases occurring in the absence of the other clinical symptoms ${ }^{116}$. This has caused considerable interest in investigating a potential role for neuronal antibodies in the pathogenesis of psychiatric syndromes. Various casecontrol studies have produced conflicting results, but a systematic review and meta-analysis found that serum NMDAR antibodies were three times as common in patients with schizophrenia, schizoaffective disorder, bipolar affective disorder or major depressive disorder compared to controls ${ }^{117,118}$. This finding was supported by a more recent large case-control study indicating that serum NMDAR antibodies were more prevalent in patients with first episode psychosis than in the healthy controls, although this was not the case for the other antibodies tested; LGI1, GABA $\mathrm{A}$ R and VGKC-complex ${ }^{119}$. However, in the psychiatric patient population, the clinical characteristics between antibody positive and antibody negative patients appear similar and to date the clinical significance of this increased prevalence is unclear ${ }^{120}$.

Cognitive impairment is a central feature of schizophrenia with the dysfunction extending across domains of memory, attention and executive function ${ }^{121}$. These deficits occur before the onset of psychosis and remain stable throughout the course of the disease ${ }^{122,123}$. Glutamate receptor hypofunction has been hypothesised to underlie this cognitive impairment and more recently NMDAR autoimmunity has been implicated. Indeed, a recent study found that first episode psychosis patients with schizophrenia who had a positive serum NMDAR antibody exhibited greater cognitive impairments in all domains relative to controls ${ }^{124}$. Furthermore, serum antibody level was inversely correlated with scores in verbal and learning memory, working memory and speed of processing. In this clinical population, the pathogenicity of NMDAR antibodies also appears to relate to blood-brain barrier integrity $^{101,125}$. Increased blood-brain barrier permeability is known to associate with Toxoplasma gondii exposure in human cohorts and NMDAR antibody seropositivity (to the NR2 subunit) in schizophrenia was associated with higher degrees of cognitive impairment where it coexisted with Toxoplasma gondii exposure ${ }^{125}$. While these findings are of great interest, no firm conclusions can be made without replication on a larger scale.

\section{Dementia}

Neuronal autoantibodies have also been detected at relatively high frequencies in a number of dementia syndromes; in one study increased prevalence of NMDAR antibodies, predominantly IgA and IgM, was demonstrated across all types of dementia $(16 \%$ vs $2 \%$ in controls $)^{126}$. While it remains unclear whether these autoantibodies have a primary pathogenic role or reflect a response to neuronal damage, there is some evidence to support a possible role in mediating cognitive symptoms. Patients with neurodegenerative disease such as Parkinson's disease have been found to have serum NMDAR antibody frequencies in the range of controls unless there is evidence of dementia e.g., dementia with Lewy bodies or Parkinson's disease dementia ${ }^{126}$. Although no such association was found in a more recent study of Parkinson's disease with dementia, given the cognitive impairment in the studied group was modest (mean MMSE 25) this merits replication ${ }^{127}$.

The prevalence of serum NMDAR antibodies is not uniformly distributed across dementia subtypes. Disproportionately high frequencies of positive antibodies $(>60 \%)$ are found in 'unclassified' or 'atypical' dementias $^{102,126}$. These patients frequently had subacute onset with rapid progression or fluctuation and an inflammatory CSF, often showing reversibility when treated with immunotherapy ${ }^{126,128}$. In a recent meta-analysis, we reported that both IgG and IgA/M serum NMDAR antibodies were more prevalent in atypical dementias vs healthy controls, while there was no difference for allcause dementia. However, the total number of studies was small and "atypicality" was inconsistently defined and in some studies may have been done so post-hoc, necessitating caution in interpretation of this intriguing result ${ }^{129}$.

The term "autoimmune dementia" has been proposed to describe this subacute cognitive impairment responsive to immunotherapy and, while its prevalence is unclear, there is growing suspicion that many cases may go undiagnosed, overlooked as primary neurodegenerative dementias ${ }^{130}$. In a study of 56 patients, a third of those who responded to immunotherapy, with notable improvements in all cognitive domains, had been initially diagnosed with a neurodegenerative or prion disorder ${ }^{131}$. Numerous case reports have illustrated the potential for antibodies to produce a phenocopy of established dementia syndromes, with misdiagnoses seen in cases of both NMDAR and LGI1 encephalitis mimicking atypical neurodegenerative dementias ${ }^{11,37}$. Given the reversible nature of autoimmune dementia, potential misdiagnoses of this nature could be catastrophic and all efforts to avoid them must be made.

\section{Neurodevelopmental implications}

Placental transfer of IgG antibodies during gestation is a well-established phenomenon, with these antibodies also having the potential to penetrate the foetal blood-brain barrier during specific developmental windows ${ }^{132,133}$. It is 
therefore perhaps unsurprising that in utero autoantibody exposure has been implicated neurodevelopmentally as a pathogenic factor altering the cognitive development of the foetus. CASPR2 is known to have a critical role in neurodevelopment, is highly expressed in the proliferating zones and is necessary for dendritic spine development and arborisation, integral to neural circuit assembly ${ }^{134}$. In CNTNAP2 knockout mice, which lack the predominant CASPR2 isoform, there are abnormalities in neuronal migration and reduced inhibitory GABAergic neurons causing a typical autistic phenotype with spontaneous seizures $^{135}$. This phenotype is mirrored in paediatric patients with homozygous CNTNAP2 mutations who lack CASPR $2^{136}$. Mice exposed to CASPR2 antibody in utero show abnormal cortical migration and development with reduced glutamatergic synapses, increased microglial activation and decreased hippocampal inhibitory neurons ${ }^{137,138}$. The offspring showed subsequent long-term behavioural sequelae with repetitive behaviour and impairments in sociability and flexible learning ${ }^{137,138}$. Prevalence of CASPR2 antibodies was markedly higher in a subgroup of mothers with autistic children (37\%) than in the control groups $(8-12 \%)^{137}$. These results were not replicated in a Danish cohort study but CASPR2 antibodies were found more frequently in the mothers of children with "mental retardation or disorders of psychological development"139. This study did not find a significant association between maternal NMDAR antibodies and child cognitive development but a murine model has demonstrated reduced density of NMDAR in neonates of mothers with NMDAR antibodies with associated neuropathological changes and greater postnatal mortality and chronic increased hyperactivity ${ }^{140}$. An association between maternal lupus, with anti-dsDNA antibodies cross-reacting with the NR2A/NR2B subunits of the NMDAR, and neurocognitive problems in the offspring has been reported, with the offspring showing deficits in behaviour, memory and learning ${ }^{141}$. dsDNAspecific NMDAR antibodies injected into pregnant dams caused thinned, disorganised cortex in offspring with subsequent cognitive impairments ${ }^{142}$. While there is no evidence of overlap with the NR1 NMDAR antibodies seen in encephalitis it illustrates the potential for these antibodies to also exert an effect on neurodevelopment ${ }^{143}$.

\section{Conclusions and future directions}

Each neuronal antibody exerts a distinct mechanistic effect and while the downstream effects all include cognitive dysfunction, the affected domains vary between subtype. However, there is much work to be done in fully characterising both the acute and chronic impairments of the encephalitic syndromes; current descriptions tend to be mostly qualitative and for the less common subtypes data is sparse.
Outside of encephalitis, pathogenicity of the neuronal antibodies may be contingent on factors including the integrity of the blood-brain barrier. Where this is compromised there is often evidence of secondary cognitive dysfunction; we postulate that blood-brain barrier disruption modulates much of the heterogeneity seen in the impact of neuronal antibodies outside of encephalitis. The site of autoantibody production may be equally important. In autoimmune encephalitis, it is likely that ongoing peripheral germinal centre reactions generate antigenspecific B cells. Subsequently, antigen-secreting cells that have differentiated from these B cells likely access the CNS, resulting in intrathecal production of pathogenic $\operatorname{IgG}^{144}$. It is not at all clear that the same process is occurring in the non-encephalitic situations described in this review. Indeed, blood-brain barrier disruption might be an important factor in some of the situations described above precisely because there is no intrathecal production of pathogenic antibodies. Furthermore, the description of unmutated yet functional (and potentially pathogenic, despite low affinity) NMDAR antibodies raises the possibility that, outside of the encephalitis context, the clinical relevance and/or pathogenicity of neuronal autoantibodies might in fact arise from the so-called 'healthy' naïve B cell repertoire ${ }^{145}$. Differences in epitope specificity, antibody titres and duration of interaction and initial immunising stimulus may also all have relevance in distinguishing encephalitis cases from non-encephalitis cases where the antibodies nonetheless may have some pathogenic role. An alternative, and underexplored, perspective is that neuronal autoantibodies (particularly "natural" antibodies with different binding properties) could have an adaptive physiological role; recent animal (and to some extent human) work suggests that NMDAR antibodies could be produced in response to stress as a mechanism to reduce anxiety/ depressive behaviours, possibly via NMDAR antagonism $^{146}$. It is conceivable that there is an analogous role in preventing, for example, excitotoxicity-mediated neuronal damage and cognitive impairment in some circumstances, although this remains to be explored.

Crucially, there is very little evidence currently for or against the possibility that immunotherapy could be an effective treatment in any of these non-encephalitis situations; the main notable exceptions are in cases of atypical or "autoimmune dementia"-and in some of these cases the demarcation from autoimmune encephalitis is far from clear ${ }^{126,128,131}$. The possibility that specific immunotherapies could have a role in treating cancerassociated cognitive impairment, or as a treatment to prevent the progression of cognitive impairment in neuronal antibody-positive post-HSV encephalitis patients, for example, warrants further evaluation.

Non-IgG NMDAR antibodies, in particular, have been implicated in the cognitive impairment seen in a 
multitude of disorders including cancer, dementia and schizophrenia. While NMDAR antibodies have been shown to universally have pathogenic potential ${ }^{94}$, the clinical consequence may vary with isotype. It is well established that the IgG isotype can cause NMDAR encephalitis while IgA NMDAR antibodies have been implicated in a more insidious cognitive impairment ${ }^{128}$. Indeed, the frequencies of NMDAR antibodies detected in dementia, cancer and stroke are more than twofold greater for IgA or IgM isotypes than $\operatorname{IgG}^{102,106,113}$. We suggest that these isotypes may be relevant for understanding cognitive impairment outside of encephalitis. While the potential exists for all the neuronal antibodies described to impact cognition, further work is needed to characterise this. In the future this could pave the way for novel, immunologically-based therapeutic options to treat cognitive impairment, with potentially transformative implications.

\section{Acknowledgements}

T.A.P. was supported in a Clinical Research Training Fellowship by the Wellcome Trust (Grant Number: 105758/Z/14/Z) and in a clinical lectureship by the National Institute for Health Research (NIHR). The views expressed are those of the author (s) and not necessarily those of the NIHR or the Department of Health and Social Care. C.F. was support by the Deutsche Forschungsgemeinschaft (DFG, German Research Foundation; grant numbers 327654276 (SFB 1315) and FI 2309/2-1) and the German Federal Ministry of Education and Research (BMBF; Grant Numbers 13GW0212A, 13GW0206D and 01GM1908D).

\section{Author details}

'Department of Old Age Psychiatry, Institute of Psychiatry, Psychology and Neuroscience, King's College London, London, UK. ${ }^{2}$ University of Cambridge, Cambridge, UK. ${ }^{3}$ Department of Basic and Clinical Neuroscience, Institute of Psychiatry, Psychology and Neuroscience, King's College London, London, UK. ${ }^{4}$ MRC Centre for Neurodevelopmental Disorders, King's College London, London, UK. ${ }^{5}$ Department of Neurology, Charité - Universitätsmedizin Berlin, Berlin, Germany. ${ }^{6}$ Berlin School of Mind and Brain, Humboldt-Universität zu Berlin, Berlin, Germany. ${ }^{7}$ Department of Psychosis Studies, Institute of

Psychiatry, Psychology and Neuroscience, King's College London, London, UK

\section{Conflict of interest}

The authors declare that they have no conflict of interest.

\section{Publisher's note}

Springer Nature remains neutral with regard to jurisdictional claims in published maps and institutional affiliations.

Received: 27 January 2020 Revised: 29 July 2020 Accepted: 30 July 2020 Published online: 01 September 2020

\section{References}

1. Schmidt-Wilcke, T. et al. GABA-from inhibition to cognition: emerging concepts. Neuroscientist 24, 501-515 (2018).

2. Sohal, V. S. \& Rubenstein, J. L. R. Excitation-inhibition balance as a framework for investigating mechanisms in neuropsychiatric disorders. Mol. Psychiatry 24, 1248-1257 (2019).

3. Zhou, S. \& Yu, Y. Synaptic E-l balance underlies efficient neural coding. Front Neurosci. 12, 46 (2018).

4. Kornau, H. C. et al. Human cerebrospinal fluid monoclonal LGl1 autoantibodies increase neuronal excitability. Ann. Neurol. 87, 405-418 (2020)
5. Kreye, J. et al. Human cerebrospinal fluid monoclonal N-methyl-D-aspartate receptor autoantibodies are sufficient for encephalitis pathogenesis. Brain. 139, 2641-2652 (2016).

6. Titulaer, M. J. et al. Treatment and prognostic factors for long-term outcome in patients with anti-NMDA receptor encephalitis: an observational cohort study. Lancet Neurol. 12, 157-165 (2013).

7. Nicolle, D. C. M. \& Moses, J. L. A systematic review of the neuropsychological sequelae of people diagnosed with anti N-methyl-D-aspartate receptor encephalitis in the acute and chronic phases. Arch. Clin. Neuropsychol. 33, 964-983 (2018).

8. McKeon, G. L. et al. Cognitive outcomes following anti-N-methyl-D-aspartate receptor encephalitis: a systematic review. J. Clin. Exp. Neuropsychol. 40, 234-252 (2018).

9. Kuroda, T. et al. Autobiographical age awareness disturbance syndrome in autoimmune limbic encephalitis: two case reports. BMC Neurol. 15, 238 (2015).

10. Savage, S. A., Irani, S. R., Leite, M. I. \& Zeman, A. Z. NMDA receptor antibody encephalitis presenting as transient epileptic amnesia. J. Neuroimmunol. 327, 41-43 (2019).

11. Abe, K. \& Chiba, Y. A case of treatable dementia with Lewy bodies remarkably improved by immunotherapy. J. Neuroimmunol. 330, 35-37 (2019).

12. Gibson, L. L. et al. The psychiatric phenotype of anti-NMDA receptor encephalitis. J. Neuropsychiatry Clin. Neurosci. 31, 70-79 (2019).

13. Finke, C. et al. Cognitive deficits following anti-NMDA receptor encephalitis. J. Neurol. Neurosurg. Psychiatry 83, 195-198 (2012).

14. McKeon, G. L. et al. Cognitive and social functioning deficits after anti-Nmethyl-D-aspartate receptor encephalitis: an exploratory case series. J. Int Neuropsychol. Soc. 22, 828-838 (2016).

15. Hughes, E. G. et al. Cellular and synaptic mechanisms of anti-NMDA receptor encephalitis. J. Neurosci. 30, 5866-5875 (2010).

16. Moscato, E. H. et al. Acute mechanisms underlying antibody effects in anti-Nmethyl-D-aspartate receptor encephalitis. Ann. Neurol. 76, 108-119 (2014).

17. Mikasova, L. et al. Disrupted surface cross-talk between NMDA and Ephrin-B2 receptors in anti-NMDA encephalitis. Brain: a J. Neurol. 135, 1606-1621 (2012).

18. Planaguma, J. et al. Ephrin-B2 prevents N-methyl-D-aspartate receptor antibody effects on memory and neuroplasticity. Ann. Neurol. 80, 388-400 (2016).

19. Zhang, Q. et al. Suppression of synaptic plasticity by cerebrospinal fluid from anti-NMDA receptor encephalitis patients. Neurobiol. Dis. 45, 610-615 (2012).

20. Wang, $X$. et al. Neuronal NMDAR currents of the hippocampus and learning performance in autoimmune anti-NMDAR encephalitis and involvement of TNF-alpha and IL-6. Front Neurol. 10, 684 (2019).

21. Kersten, M. et al. Novel object recognition in rats with NMDAR dysfunction in CA1 after stereotactic injection of anti-NMDAR encephalitis cerebrospinal fluid. Front Neurol. 10, 586 (2019).

22. Moghaddam, B., Adams, B., Verma, A. \& Daly, D. Activation of glutamatergic neurotransmission by ketamine: a novel step in the pathway from NMDA receptor blockade to dopaminergic and cognitive disruptions associated with the prefrontal cortex. J. Neurosci. 17, 2921-2927 (1997).

23. Monaghan, D. T., Yao, D. \& Cotman, C. W. L-[3H]Glutamate binds to kainate-, NMDA- and AMPA-sensitive binding sites: an autoradiographic analysis. Brain Res 340, 378-383 (1985).

24. Finke, C. et al. Functional and structural brain changes in anti-N-methyl-Daspartate receptor encephalitis. Ann. Neurol. 74, 284-296 (2013).

25. Peer, $M$. et al. Functional connectivity of large-scale brain networks in patients with anti-NMDA receptor encephalitis: an observational study.Lancet Psychiatry 4, 768-774 (2017).

26. Finke, C. et al. Structural hippocampal damage following anti-N-methyl-Daspartate receptor encephalitis. Biol. Psychiatry 79, 727-734 (2016).

27. Phillips, O. R. et al. Superficial white matter damage in anti-NMDA receptor encephalitis. J. Neurol. Neurosurg. Psychiatry 89, 518-525 (2018).

28. Jones, B. E. et al. Autoimmune receptor encephalitis in mice induced by active immunization with conformationally stabilized holoreceptors. Sci. Transl. Med. 11, eaaw0044 (2019).

29. Buckley, C. et al. Potassium channel antibodies in two patients with reversible limbic encephalitis. Ann. Neurol. 50, 73-78 (2001).

30. Irani, S. R. et al. Antibodies to Kv1 potassium channel-complex proteins leucine-rich, glioma inactivated 1 protein and contactin-associated protein-2 
in limbic encephalitis, Morvan's syndrome and acquired neuromyotonia. Brain 133, 2734-2748 (2010).

31. Lang, B. et al. Intracellular and non-neuronal targets of voltage-gated potassium channel complex antibodies. J. Neurol. Neurosurg. Psychiatry $\mathbf{8 8}$ 353-361 (2017).

32. van Sonderen, A. et al. The relevance of VGKC positivity in the absence of LGl1 and Caspr2 antibodies. Neurology 86, 1692-1699 (2016).

33. Irani, S. R. et al. Faciobrachial dystonic seizures precede Lgi1 antibody limbic encephalitis. Ann. Neurol. 69, 892-900 (2011).

34. Binks, S. N. M. et al. LGI1, CASPR2 and related antibodies: a molecular evolution of the phenotypes. J. Neurol. Neurosurg. Psychiatry 89, 526-534 (2018).

35. Arino, H. et al. Anti-LGI1-associated cognitive impairment: presentation and long-term outcome. Neurology 87, 759-765 (2016).

36. Joubert, B. et al. Characterization of a subtype of autoimmune encephalitis with anti-contactin-associated protein-like 2 antibodies in the cerebrospinal fluid, prominent limbic symptoms, and seizures. JAMA Neurol. 73, 1115-1124 (2016).

37. Li, X., Yuan, J., Liu, L. \& Hu, W. Antibody-LGI 1 autoimmune encephalitis manifesting as rapidly progressive dementia and hyponatremia: a case report and literature review. BMC Neurol. 19, 19 (2019).

38. Marquetand, J. et al. Slowly progressive LGI1 encephalitis with isolated lateonset cognitive dysfunction: a treatable mimic of Alzheimer's disease. Eur. J. Neurol. 23, e28-e29 (2016).

39. Butler, C. R. et al. Persistent anterograde amnesia following limbic encephalitis associated with antibodies to the voltage-gated potassium channel complex. J. Neurol. Neurosurg. Psychiatry 85, 387-391 (2014).

40. Finke, C. et al. Evaluation of cognitive deficits and structural hippocampal damage in encephalitis with leucine-rich, glioma-inactivated 1 antibodies. JAMA Neurol. 74, 50-59 (2017).

41. Hanert, A. et al. Hippocampal dentate gyrus atrophy predicts pattern separation impairment in patients with LGl1 encephalitis. Neuroscience 400, 120-131 (2019).

42. van Sonderen, A. et al. Anti-LGl1 encephalitis: clinical syndrome and longterm follow-up. Neurology 87, 1449-1456 (2016).

43. Thompson, J. et al. The importance of early immunotherapy in patients with faciobrachial dystonic seizures. Brain: a J. Neurol. 141, 348-356 (2018).

44. Irani, S. R. et al. Faciobrachial dystonic seizures: the influence of immunotherapy on seizure control and prevention of cognitive impairment in a broadening phenotype. Brain 136, 3151-3162 (2013).

45. Ohkawa, T. et al. Autoantibodies to epilepsy-related LGl1 in limbic encephalitis neutralize LGI1-ADAM22 interaction and reduce synaptic AMPA receptors. J. Neurosci. 33, 18161-18174 (2013).

46. Lalic, T., Pettingill, P., Vincent, A. \& Capogna, M. Human limbic encephalitis serum enhances hippocampal mossy fiber-CA3 pyramidal cell synaptic transmission. Epilepsia 52, 121-131 (2011).

47. Petit-Pedrol, M. et al. LGI1 antibodies alter Kv1.1 and AMPA receptors changing synaptic excitability, plasticity and memory. Brain 141, 3144-3159 (2018).

48. Ramberger, M. et al. Distinctive binding properties of human monoclonal LGI1 autoantibodies determine pathogenic mechanisms. Brain I Neurol. https://doi.org/10.1093/brain/awaa104 (2020).

49. Kalachikov, S. et al. Mutations in LGI1 cause autosomal-dominant partial epilepsy with auditory features. Nat. Genet. 30, 335-341 (2002).

50. Herranz-Perez, V., Olucha-Bordonau, F. E., Morante-Redolat, J. M. \& Perez-Tur, J. Regional distribution of the leucine-rich glioma inactivated (LGI) gene family transcripts in the adult mouse brain. Brain Res. 1307, 177-194 (2010).

51. Vincent, A. et al. Potassium channel antibody-associated encephalopathy: a potentially immunotherapy-responsive form of limbic encephalitis. Brain 127, 701-712 (2004).

52. Kotsenas, A. L. et al. MRI findings in autoimmune voltage-gated potassium channel complex encephalitis with seizures: one potential etiology for mesial temporal sclerosis. Am. J. Neuroradiol. 35, 84-89 (2014).

53. Miller, T. D. et al. Focal CA3 hippocampal subfield atrophy following LGI1 VGKC-complex antibody limbic encephalitis. Brain 140, 1212-1219 (2017).

54. Loane, C. et al. Hippocampal network abnormalities explain amnesia after VGKCC-Ab related autoimmune limbic encephalitis. J. Neurol. Neurosurg. Psychiatry 90, 965-974 (2019).

55. Heine, J. et al. Beyond the limbic system: disruption and functional compensation of large-scale brain networks in patients with anti-LGl1 encephalitis. J. Neurol. Neurosurg. Psychiatry 89, 1191-1199 (2018).
56. Saint-Martin, M. et al. Contactin-associated protein-like 2, a protein of the neurexin family involved in several human diseases. Eur. J. Neurosci. 48, 1906-1923 (2018).

57. Irani, S. R. et al. Morvan syndrome: clinical and serological observations in 29 cases. Ann. Neurol. 72, 241-255 (2012).

58. Armangue, T. et al. Frequency, symptoms, risk factors, and outcomes of autoimmune encephalitis after herpes simplex encephalitis: a prospective observational study and retrospective analysis. Lancet Neurol. 17, 760-772 (2018).

59. Poliak, S. et al. Juxtaparanodal clustering of Shaker-like $\mathrm{K}+$ channels in myelinated axons depends on Caspr2 and TAG-1. J. Cell Biol. 162, 1149-1160 (2003).

60. Varea, O. et al. Synaptic abnormalities and cytoplasmic glutamate receptor aggregates in contactin associated protein-like 2/Caspr2 knockout neurons. Proc. Natl Acad. Sci. USA 112, 6176-6181 (2015).

61. Fernandes, D. et al. Disrupted AMPA receptor function upon genetic- or antibody-mediated loss of autism-associated CASPR2. Cereb. cortex 29, 4919-4931 (2019).

62. Pinatel, D. et al. Inhibitory axons are targeted in hippocampal cell culture by anti-Caspr2 autoantibodies associated with limbic encephalitis. Front Cell Neurosci. 9, 265 (2015).

63. Saint-Martin, M. et al. Impact of anti-CASPR2 autoantibodies from patients with autoimmune encephalitis on CASPR2/TAG-1 interaction and Kv1 expression. J. Autoimmun. 103, 102284 (2019).

64. Olsen, A. L. et al. Caspr2 autoantibodies target multiple epitopes. Neurol.(R.) Neuroimmunol. Neuroinflammation 2, e127 (2015).

65. Patterson, K. R., Dalmau, J. \& Lancaster, E. Mechanisms of Caspr2 antibodies in autoimmune encephalitis and neuromyotonia. Ann. Neurol. 83, 40-51 (2018).

66. Giannoccaro, M. P. et al. Behaviour and neuropathology in mice injected with human contactin-associated protein 2 antibodies. Brain 142, 2000-2012 (2019).

67. Fernandes, D. et al. Disrupted AMPA Receptor Function upon Genetic- or Antibody-Mediated Loss of Autism-Associated CASPR2. Cereb Cortex, https:// doi.org/10.1093/cercor/bhz032 (2019).

68. Hoftberger, R. et al. Encephalitis and AMPA receptor antibodies: novel findings in a case series of 22 patients. Neurology 84, 2403-2412 (2015).

69. Lai, M. et al. AMPA receptor antibodies in limbic encephalitis alter synaptic receptor location. Ann. Neurol. 65, 424-434 (2009).

70. Joubert, B. et al. Clinical spectrum of encephalitis associated with antibodies against the alpha-amino-3-hydroxy-5-methyl-4-isoxazolepropionic acid receptor: case series and review of the literature. JAMA Neurol. 72, 1163-1169 (2015).

71. Laurido-Soto, O. et al. Patient characteristics and outcome associations in AMPA receptor encephalitis. J. Neurol. 266, 450-460 (2019).

72. Samad, N. \& Wong, J. Anti-AMPA receptor encephalitis associated with medullary thyroid cancer. BMJ Case Rep 2018, bcr2018225745. https:/doi. org/10.1136/bcr-2018-225745 (2018).

73. Spatola, M. et al. Serial brain (1)(8)FDG-PET in anti-AMPA receptor limbic encephalitis. J. Neuroimmunol. 271, 53-55 (2014).

74. Henley, J. M. \& Wilkinson, K. A. AMPA receptor trafficking and the mechanisms underlying synaptic plasticity and cognitive aging. Dialogues Clin. Neurosci. 15, 11-27 (2013).

75. Gleichman, A. J. et al. Antigenic and mechanistic characterization of antiAMPA receptor encephalitis. Ann. Clin. Transl. Neurol. 1, 180-189 (2014).

76. Peng, X. et al. Cellular plasticity induced by anti-alpha-amino-3-hydroxy-5methyl-4-isoxazolepropionic acid (AMPA) receptor encephalitis antibodies. Ann. Neurol. 77, 381-398 (2015).

77. Haselmann, $\mathrm{H}$. et al. Human autoantibodies against the AMPA receptor subunit GluA2 induce receptor reorganization and memory dysfunction. Neuron 100, 91-105. e109 (2018).

78. Granger, A. J. et al. LTP requires a reserve pool of glutamate receptors independent of subunit type. Nature 493, 495-500 (2013).

79. Wei, Y. C. et al. Rapid progression and brain atrophy in anti-AMPA receptor encephalitis. J. Neuroimmunol. 261, 129-133 (2013).

80. Petit-Pedrol, M. et al. Encephalitis with refractory seizures, status epilepticus, and antibodies to the GABAA receptor: a case series, characterisation of the antigen, and analysis of the effects of antibodies. Lancet Neurol. 13, 276-286 (2014).

81. Spatola, M. et al. Investigations in GABAA receptor antibody-associated encephalitis. Neurology 88, 1012-1020 (2017). 
82. Pettingill, $P$. et al. Antibodies to GABAA receptor alpha1 and gamma2 subunits: clinical and serologic characterization. Neurology $\mathbf{8 4}$ 1233-1241 (2015).

83. Nikolaus, M. et al. Severe GABAA receptor encephalitis without seizures: a paediatric case successfully treated with early immunomodulation. European journal of paediatric neurology: EJPN: official journal of the European Paediatric Neurology. Society 22, 558-562 (2018).

84. O'Connor, K. et al. GABAA receptor autoimmunity: a multicenter experience. Neurol.(R.) Neuroimmunol. neuroinflammation 6, e552 (2019).

85. Ohkawa, T. et al. Identification and characterization of GABA(A) receptor autoantibodies in autoimmune encephalitis. J. Neurosci. 34, 8151-8163 (2014).

86. Macdonald, R. L., Kang, J. Q. \& Gallagher, M. J. Mutations in GABAA receptor subunits associated with genetic epilepsies. J. Physiol. 588, 1861-1869 (2010).

87. Nikolaus, $M$. et al. CSF reactivity in GABAA receptor antibody encephalitis-Immunocytochemical distribution in the murine brain. Brain Res. 1704, 249-256 (2019).

88. Lancaster, E. et al. Antibodies to the GABA(B) receptor in limbic encephalitis with seizures: case series and characterisation of the antigen. Lancet Neurol. $\mathbf{9}$, 67-76 (2010).

89. Hoftberger, R. et al. Encephalitis and GABAB receptor antibodies: novel findings in a new case series of 20 patients. Neurology 81, 1500-1506 (2013).

90. van Coevorden-Hameete, M. H. et al. The expanded clinical spectrum of antiGABABR encephalitis and added value of KCTD16 autoantibodies. Brain: a J. Neurol. 142, 1631-1643 (2019).

91. Nibber, A. et al. Pathogenic potential of antibodies to the GABAB receptor. Epilepsia Open 2, 355-359 (2017).

92. Schuler, V. et al. Epilepsy, hyperalgesia, impaired memory, and loss of preand postsynaptic $G A B A(B)$ responses in mice lacking $G A B A(B(1))$. Neuron 31 47-58 (2001).

93. Cui, J. et al. The gamma-aminobutyric acid-B receptor (GABAB) encephalitis: clinical manifestations and response to immunotherapy. Int. J. Neurosci. 128 627-633 (2018).

94. Castillo-Gomez, E. et al. All naturally occurring autoantibodies against the NMDA receptor subunit NR1 have pathogenic potential irrespective of epitope and immunoglobulin class. Mol. Psychiatry 22, 1776-1784 (2017).

95. Ehrenreich, $\mathrm{H}$. Autoantibodies against $\mathrm{N}$-methyl-d-aspartate receptor 1 in health and disease. Curr. Opin. Neurol. 31, 306-312 (2018).

96. Dahm, L. et al. Seroprevalence of autoantibodies against brain antigens in health and disease. Ann. Neurol. 76, 82-94 (2014).

97. Jezequel, J. et al. Cell- and single molecule-based methods to detect anti-Nmethyl-D-aspartate receptor autoantibodies in patients with first-episode psychosis from the OPTiMiSE project. Biol. Psychiatry 82, 766-772 (2017).

98. Zandi, M. S. et al. Clinical relevance of serum antibodies to extracellular Nmethyl-d-aspartate receptor epitopes. J. Neurol. Neurosurg. Psychiatry $\mathbf{8 6}$, 708-713 (2015).

99. Graus, F. et al. A clinical approach to diagnosis of autoimmune encephalitis. Lancet Neurol. 15, 391-404 (2016).

100. Pollak, T. A. et al. Autoimmune psychosis: an international consensus on an approach to the diagnosis and management of psychosis of suspected autoimmune origin. Lancet Psychiatry 7, 93-108 (2020).

101. Hammer, C. et al. Neuropsychiatric disease relevance of circulating antiNMDA receptor autoantibodies depends on blood-brain barrier integrity. Mol. Psychiatry 19, 1143-1149 (2014).

102. Busse, M. et al. Dysfunction of the blood-cerebrospinal fluid-barrier and $\mathrm{N}$ methyl-D-aspartate glutamate receptor antibodies in dementias. Eur. Arch. Psychiatry Clin. Neurosci. 268, 483-492 (2018).

103. Levin, E. C. et al. Brain-reactive autoantibodies are nearly ubiquitous in human sera and may be linked to pathology in the context of blood-brain barrier breakdown. Brain Res. 1345, 221-232 (2010).

104. Kowal, C. et al. Human lupus autoantibodies against NMDA receptors mediate cognitive impairment. Proc. Natl Acad. Sci. USA 103, 19854-19859 (2006)

105. Dalmau, J., Geis, C. \& Graus, F. Autoantibodies to synaptic receptors and neuronal cell surface proteins in autoimmune diseases of the central nervous system. Physiol. Rev. 97, 839-887 (2017).

106. Finke, C. et al. High prevalence of neuronal surface autoantibodies associated with cognitive deficits in cancer patients. J. Neurol. 264, 1968-1977 (2017).

107. Bartels, F. et al. Neuronal autoantibodies associated with cognitive impairment in melanoma patients. Ann. Oncol. 30, 823-829 (2019).
108. Armangue, T. et al. Herpes simplex virus encephalitis is a trigger of brain autoimmunity. Ann. Neurol. 75, 317-323 (2014).

109. Pruss, H. Postviral autoimmune encephalitis: manifestations in children and adults. Curr. Opin. Neurol. 30, 327-333 (2017).

110. Salovin, A. et al. Anti-NMDA receptor encephalitis and nonencephalitic HSV-1 infection. Neurol. Neuroimmunol. Neuroinflammation 5, e458 (2018).

111. Dale, R. C. \& Nosadini, M. Infection-triggered autoimmunity: The case of herpes simplex virus type 1 and anti-NMDAR antibodies. Neurol. Neuroimmunol. Neuroinflammation 5, e471 (2018).

112. Westman, G. et al. N-Methyl-D-Aspartate receptor Autoimmunity Affects Cognitive Performance in herpes simplex encephalitis. Clin. Microbiol. Infect, https://doi.org/10.1016/j.cmi.2016.07.028 (2016).

113. Zerche, $M$. et al. Preexisting serum autoantibodies against the NMDAR subunit NR1 modulate evolution of lesion size in acute ischemic. Stroke Stroke 46, 1180-1186 (2015).

114. Royl, G. et al. Antibodies against neural antigens in patients with acute stroke: joint results of three independent cohort studies. J. Neurol. 266, 2772-2779 (2019).

115. Sperber, P. S. et al. Serum anti-NMDA (N-methyl-D-aspartate)-receptor antibodies and long-term clinical outcome after stroke (PROSCIS-B). Stroke $\mathbf{5 0}$ 3213-3219 (2019).

116. Kayser, M. S., Titulaer, M. J., Gresa-Arribas, N. \& Dalmau, J. Frequency and characteristics of isolated psychiatric episodes in anti-N-methyl-d-aspartate receptor encephalitis. JAMA Neurol. 70, 1133-1139 (2013).

117. Hoffmann, C. et al. Absence of Autoantibodies Against Neuronal Surface Antigens in Sera of Patients With Psychotic Disorders. JAMA Psychiatry, https://doi.org/10.1001/jamapsychiatry.2019.3679 (2019).

118. Pearlman, D. M. \& Najjar, S. Meta-analysis of the association between Nmethyl-d-aspartate receptor antibodies and schizophrenia, schizoaffective disorder, bipolar disorder, and major depressive disorder. Schizophr. Res. 157 249-258 (2014).

119. Lennox, B. R. et al. Prevalence and clinical characteristics of serum neuronal cell surface antibodies in first-episode psychosis: a case-control study. Lancet Psychiatry 4, 42-48 (2017).

120. Schou, M \& Saether, S. G. NMDA receptor antibodies are found in a small subgroup of patients with first-episode psychosis, but their clinical relevance is unknown. Evid. Based Ment. Health 21, e1-e2 (2018).

121. Bowie, C. R. \& Harvey, P. D. Treatment of cognitive deficits in schizophrenia. Curr. Opin. Investig. Drugs 7, 608-613 (2006).

122. Bilder, R. M. et al. Neuropsychology of first-episode schizophrenia: initial characterization and clinical correlates. Am. J. Psychiatry 157, 549-559 (2000).

123. Rund, B. R. A review of longitudinal studies of cognitive functions in schizophrenia patients. Schizophr. Bull. 24, 425-435 (1998).

124. Tong, J. et al. Elevated serum anti-NMDA receptor antibody levels in firstepisode patients with schizophrenia. Brain Behav. Immun. 81, 213-219 (2019).

125. Kannan, G. et al. Pathogen-mediated NMDA receptor autoimmunity and cellular barrier dysfunction in schizophrenia. Transl. Psychiatry 7, e1186 (2017).

126. Doss, S. et al. High prevalence of NMDA receptor lgA/lgM antibodies in different dementia types. Ann. Clin. Transl. Neurol. 1, 822-832 (2014).

127. Hopfner, F. et al. No association between Parkinson disease and autoantibodies against NMDA-type glutamate receptors. Transl. Neurodegener. 8 , 11 (2019).

128. Pruss, $\mathrm{H}$. et al. IgA NMDA receptor antibodies are markers of synaptic immunity in slow cognitive impairment. Neurology 78, 1743-1753 (2012).

129. Gibson, L. L et al. Neuronal surface autoantibodies in dementia: a systematic review and meta-analysis. J. Neurol. https://doi.org/10.1007/s00415-02009825-0 (2020).

130. Flanagan, E. P., Drubach, D. A. \& Boeve, B. F. Autoimmune dementia and encephalopathy. Handb. Clin. Neurol. 133, 247-267 (2016).

131. Flanagan, E. P. et al. Autoimmune dementia: clinical course and predictors of immunotherapy response. Mayo Clin. Proc. 85, 881-897 (2010).

132. Braniste, $\vee$. et al. The gut microbiota influences blood-brain barrier permeability in mice. Sci. Transl. Med. 6, 263 ra158 (2014).

133. Palmeira, P. et al. lgG placental transfer in healthy and pathological pregnancies. Clin. Dev. Immunol. 2012, 985646 (2012).

134. Anderson, G. R. et al. Candidate autism gene screen identifies critical role for cell-adhesion molecule CASPR2 in dendritic arborization and spine development. Proc. Natl Acad. Sci. USA 109, 18120-18125 (2012).

135. Penagarikano, O. et al. Absence of CNTNAP2 leads to epilepsy, neuronal migration abnormalities, and core autism-related deficits. Cell 147, 235-246 (2011). 
136. Rodenas-Cuadrado, P., Ho, J. \& Vernes, S. C. Shining a light on CNTNAP2: complex functions to complex disorders. Eur. J. Hum. Genet. 22, 171-178 (2014).

137. Brimberg, L. et al. Caspr2-reactive antibody cloned from a mother of an ASD child mediates an ASD-like phenotype in mice. Mol. Psychiatry 21, 1663-1671 (2016).

138. Coutinho, E. et al. Persistent microglial activation and synaptic loss with behavioral abnormalities in mouse offspring exposed to CASPR2-antibodies in utero. Acta Neuropathologica 134, 567-583 (2017).

139. Coutinho, E. et al. CASPR2 autoantibodies are raised during pregnancy in mothers of children with mental retardation and disorders of psychological development but not autism. J. Neurol. Neurosurg. Psychiatry 88, 718-721 (2017).

140. Jurek, B. et al. Human gestational N-methyl-d-aspartate receptor autoantibodies impair neonatal murine brain function. Ann. Neurol. 86, 656-670 (2019).

141. Urowitz, M. B. et al. Neurocognitive abnormalities in offspring of mothers with systemic lupus erythematosus. Lupus 17, 555-560 (2008).
142. Lee, J. Y. et al. Neurotoxic autoantibodies mediate congenital cortical impairment of offspring in maternal lupus. Nat. Med. 15, 91-96 (2009).

143. Hirohata, S. \& Tanaka, K. Differential expression of antibodies to NMDA receptor in anti-NMDA receptor encephalitis and in neuropsychiatric systemic lupus erythematosus. Lupus Sci. Med. 6, e000359 (2019).

144. Makuch, M. et al. N-methyl-D-aspartate receptor antibody production from germinal center reactions: Therapeutic implications. Ann. Neurol. 83, 553-561 (2018).

145. Wenke, N. K et al. N-methyl-D-aspartate receptor dysfunction by unmutated human antibodies against the NR1 subunit. Ann. Neurol. 85 771-776 (2019).

146. Pan, $H$. et al. Multiple inducers and novel roles of autoantibodies against the obligatory NMDAR subunit NR1: a translational study from chronic life stress to brain injury. Mol. Psychiatry, https://doi.org/10.1038/s41380-020-0672-1 (2020).

147. Dalmau, J. \& Graus, F. Antibody-mediated encephalitis. N. Engl. J. Med. 378, 840-851 (2018). 